\title{
The population of bright NEAs
}

\author{
G. B. Valsecchi ${ }^{1,2}$ and G. F. Gronchi ${ }^{3}$ \\ ${ }^{1}$ IAPS-INAF, Roma, Italy \\ ${ }^{2}$ IFAC-CNR, Sesto Fiorentino, Italy \\ ${ }^{3}$ University of Pisa, Pisa, Italy
}

Our understanding of the orbital distribution of NEAs is influenced by discovery selection effects, so that it is likely that the orbital distribution of known NEAs differs from the true distribution. In fact, our ability to reconstruct the true distribution critically depends on the removal of discovery biases from the known population.

The reference model of the orbital distribution of NEAs is described in Bottke et al. (2002). It combines dynamics (a numerical reconstruction of how NEAs are transferred from source regions to their current orbits), with the observational record of Spacewatch, whose biases are well studied (Jedicke 1996). According to Bottke et al., the proportion of orbital classes within the NEA population with $H \leqslant 18$ should be $32 \% \pm 1 \%$ Amors, $62 \% \pm 1 \%$ Apollos, $6 \% \pm 1 \%$ Atens, and $2 \% \pm 0 \%$ IEOs; moreover, the fraction of PHAs (i.e., objects with $M O I D \leqslant 0.05 \mathrm{AU}$ ) should be $21 \%$, and Bottke et al. give also the proportions, within the various subclasses, of objects with $a<2 \mathrm{AU}, e<0.4, e<0.6$, $i<10^{\circ}, i<20^{\circ}$ and $i<30^{\circ}$.

About 1500 NEAs were known when the NEA population model just discussed was developed, and the estimated completeness for $H \leqslant 18$ was about $50 \%$. More than 9000 NEAs are known now, the estimated completeness for $H \leqslant 18$ is much higher than $50 \%$, and that for $H \leqslant 16$ is very close to $100 \%$ (Harris 2011, personal communication). Because of its near-completeness, the population of NEAs with $H \leqslant 16$ is nearly unbiased; it comprised, in April 2011, 192 objects (source: http://newton.dm.unipi.it/neodys2/), a number large enough to allow for an overall statistical analysis. The orbital elements of these NEAs are in general rather well determined $\left(\sigma(a)<10^{-5} \mathrm{AU}\right)$, with very few exceptions.

Our main working hypothesis is that, since we know practically all the NEAs with $H \leqslant 16$, their orbital distribution can be considered as representative of the true orbital distribution of NEAs; with this in mind, in Table 1 we compare them to the model.

\begin{tabular}{l|r|r} 
Class & Bottke et al. & Bright NEAs \\
\hline Amors & $32 \%$ & $50 \%$ \\
Apollos & $62 \%$ & $47 \%$ \\
Atens & $6 \%$ & $3 \%$ \\
IEOs & $2 \%$ & $0 \%$ \\
PHAs & $21 \%$ & $15 \%$ \\
\hline
\end{tabular}

Table 1. The proportions of the various orbital classes among bright NEAs differs from those of Bottke et al. (2002).

Bright NEAs exhibit a significantly larger proportion of Amors, and significantly smaller proportions of Apollos, Atens, IEOs and PHAs.

As evidenced in Table 2, also the orbital distribution of bright NEAs within the classes differs from Bottke et al. (2002). As the Table shows, bright NEAs exhibit significantly larger proportions of Amors and Apollos in high-inclination orbits. 


\begin{tabular}{|c|c|c|c|c|}
\hline $\begin{array}{l}\text { Orbital } \\
\text { region }\end{array}$ & $\begin{array}{c}\text { Am } \\
\text { Bottke et al. }\end{array}$ & $\begin{array}{l}\text { hors } \\
\text { Bright NEAs }\end{array}$ & $\mid \begin{array}{c}\text { Apc } \\
\text { Bottke et al. }\end{array}$ & Bright NEAs \\
\hline$a<2 \mathrm{AU}$ & $27 \%$ & $26 \%$ & $55 \%$ & $49 \%$ \\
\hline$e<0.4$ & $25 \%$ & $23 \%$ & $9 \%$ & $9 \%$ \\
\hline$e<0.6$ & $87 \%$ & $91 \%$ & $34 \%$ & $37 \%$ \\
\hline$i<10^{\circ}$ & $41 \%$ & $25 \%$ & $20 \%$ & $16 \%$ \\
\hline$i<20^{\circ}$ & $74 \%$ & $49 \%$ & $48 \%$ & $38 \%$ \\
\hline$i<30^{\circ}$ & $87 \%$ & $72 \%$ & $67 \%$ & $59 \%$ \\
\hline
\end{tabular}

Table 2. The orbital distributions of bright NEAs differ from those of Bottke et al. (2002).

NEAs move in chaotic orbits, allowing them to encounter planets. However, this only happens for small values of the MOID relative to a given planet. Unless encounters with Jupiter are possible, encounters with the terrestrial planets able to significantly alter the orbit are infrequent, so that in the time interval between such encounters the osculating orbital elements undergo significant variations due to secular perturbations. Thus, it is of interest to consider the evolution of a NEA sample subject only to secular perturbations. Under the action of the latter, NEAs with $a>1.3 \mathrm{AU}$ may evolve to orbits with $q>1.3 \mathrm{AU}$, while some asteroids with $q>1.3 \mathrm{AU}$ may evolve to orbits with $q<1.3$ AU.

We therefore augmented our sample of bright NEAs by including the numbered and multi-opposition asteroids with $H \leqslant 16$ that can, due to secular perturbations, become NEAs (source: http://hamilton.dm.unipi.it/astdys2/). This sample, composed of bright NEAs and Potential NEAs (PNEAs), amounts to 277 objects. Its secular evolution has then been computed over a time span of $200000 \mathrm{yr}$, starting from the present epoch, with the method of Gronchi and Milani (2001). It must be kept in mind that the secular propagator we used is not appropriate for NEAs in low-order resonances.

The result of the secular integration is that the proportions within the Apollo and Amor classes given for the bright NEAs in Table 2 are remain essentially constant in time, showing that the sample seems to be in a steady state, at least from the point of view of secular perturbations. Also the proportion of PHAs remains constant.

We can thus conclude that:

- the orbital distribution of bright NEAs $(H \leqslant 16)$ is significantly different from the model by Bottke et al. (2002);

- the differences give interesting hints about the distribution of the real population: there should be more Amors, more high-inclination orbits, less PHAs compared to the model;

- for the sample of known bright NEAs, the action of secular perturbations does not significantly alter the proportions of orbital types and of PHAs.

\section{References}

Bottke, W. F., Morbidelli, A., Jedicke, R., Petit, J.-M., Levison, H. F., Michel, P., \& Metcalfe, T. S. 2002, Icarus 156,399

Gronchi, G. F. \& Milani, A. 2001, Icarus 152, 58

Jedicke, R. 1996, AJ 111, 970 